Real Analysis Exchange

Vol. 2(2), 2002/2001, pp. 811-826

Miklós Laczkovich, Department of Analysis, Eötvös University, Budapest, Kecskeméti u. 12, Hungary 1053. e-mail: laczko@renyi.hu

\title{
INFINITE PEANO DERIVATIVES
}

\begin{abstract}
Let $f_{(n)}$ and $f_{(n)}$ denote the $n^{\text {th }}$ Peano derivative and the $n^{\text {th }}$ lower Peano derivative of the function $f:[a, b] \rightarrow \mathbb{R}$. We investigate the validity of the following statements.

$\left(M_{n}\right)$. If the set $H=\left\{x \in[a, b]: f_{(n)}(x)>0\right\}$ is of positive outer measure, then $f$ is $n$-convex on a subset of $H$ having positive outer measure.

$\left(Z_{n}\right)$. The set $E_{n}(f)=\left\{x \in[a, b]: f_{(n)}(x)=\infty\right\}$ is of measure zero for every $f:[a, b] \rightarrow \mathbb{R}$.

We prove that $\left(M_{n}\right)$ and $\left(Z_{n}\right)$ are true for $n=1$ and $n=2$, but false for $n \geq 3$. More precisely we show that for every $n \geq 3$ there is an $(n-1)$ times continuously differentiable function $f$ on $[a, b]$ such that $f_{(n)}(x)=\infty$ a.e. on $[a, b]$, and that such a function cannot be $n$-convex on any set of positive outer measure.

We also show that the category analogue of $\left(Z_{n}\right)$ is false for every $n$. Moreover, the set $E_{n}(f)$ can be residual. On the other hand, the category analogue of $\left(M_{n}\right)$ is true for every $n$. More precisely, if $\{x \in$ $\left.[a, b]: f_{(n)}(x)>0\right\}$ is of second category, then $f$ is $n$-convex on a subinterval of $[a, b]$. As a corollary we find that $E_{n}(f)$ cannot be residual and of full measure simultaneously.
\end{abstract}

\section{Introduction}

Let $f^{\prime}$ denote the lower derivative of the function $f:[a, b] \rightarrow \mathbb{R}$, and let $\lambda$ denote Lebesgue outer measure. Our starting point is the following simple fact.

$\left(M_{1}\right)$. If the set $H=\left\{x \in[a, b]: f^{\prime}(x)>0\right\}$ is of positive outer measure, then there is a subset $A \subset H$ such that $\lambda(A)>0$ and $f$ is increasing on $A$.

Key Words: Peano derivatives, $n$-convex functions

Mathematical Reviews subject classification: 26A24

Received by the editors October 30, 2000 
Proof. Let $H_{n}=\left\{x \in H: \frac{f(y)-f(x)}{y-x}>0\right.$ for every $\left.0<|y-x|<1 / n\right\}$. Since $H=\cup_{n=1}^{\infty} H_{n}$, there is an index $n$ such that $\lambda\left(H_{n}\right)>0$. For a suitable $k$ the set $A=H_{n} \cap\left(\frac{k-1}{n}, \frac{k}{n}\right)$ is of positive outer measure, and it is clear that $f$ is increasing on $A$.

The following statement is also well-known.

$\left(Z_{1}\right)$. The set $\left\{x \in[a, b]: f^{\prime}(x)=\infty\right\}$ is of measure zero for every $f:[a, b] \rightarrow$ $\mathbb{R}$.

In this note we shall investigate the generalizations of the statements $\left(M_{1}\right)$ and $\left(Z_{1}\right)$ involving the notions of Peano derivatives and convexity of higher order. Let $f$ be continuous at $x$, and suppose that there exists a polynomial $p$ such that $f(x+t)=p(t)+o\left(t^{n}\right)(t \rightarrow 0)$. Then the number $p^{(k)}(0)$ is called the $k^{\text {th }}$ Peano derivative of $f$ at $x$ and is denoted by $f_{(k)}(x)$ for every $0 \leq k \leq n$. It is easy to see that $f_{(n)}(x)$ equals the limit

$$
\lim _{t \rightarrow 0} \frac{n !}{t^{n}}\left(f(x+t)-\sum_{i=0}^{n-1} \frac{f_{(i)}(x)}{i !} t^{i}\right) .
$$

If the limit (1) equals infinity, then we write $f_{(n)}(x)=\infty$. Replacing the limit by liminf in (1) we obtain the $n^{\text {th }}$ lower Peano derivative of $f$ at $x$, denoted by $\underline{f}_{(n)}(x)$. Note that $f_{(n)}(x)$ or $\underline{f}_{(n)}(x)$ are defined only if $f_{(n-1)}(x)$ exists and is finite.

The unilateral derivates $f_{(n)+}(x)$ and $f_{(n)-}(x)$ are defined by taking the corresponding unilateral limits. We shall also consider the derivatives $f_{(n)}(x)$ and $f_{(n)}(x)$ in the case when $f$ is defined on a set $A$ and $x \in A$ is a limit point of $A$. The definitions are the same except that all the limit relations have to be restricted to the set $A$.

Let $f$ be a real valued function defined on the set $A \subset \mathbb{R}$. The divided differences of $f$ are defined by induction as follows. Let $\left[x_{1} ; f\right]=f\left(x_{1}\right)$ for every $x_{1} \in A$. If $n \geq 1$ and $\left[x_{1}, \ldots, x_{n} ; f\right]$ is defined whenever $x_{1}, \ldots, x_{n}$ are distinct elements of $A$, then we put

$$
\left[x_{1}, \ldots, x_{n}, x_{n+1} ; f\right]=\frac{\left[x_{2}, \ldots, x_{n+1} ; f\right]-\left[x_{1}, \ldots, x_{n} ; f\right]}{x_{n+1}-x_{1}}
$$

for every system of distinct points $x_{1}, \ldots, x_{n+1} \in A$. An easy computation shows that

$$
\left[x_{1}, \ldots, x_{n} ; f\right]=\sum_{i=1}^{n} \frac{f\left(x_{i}\right)}{\prod_{j \neq i}\left(x_{i}-x_{j}\right)}
$$


and thus $\left[x_{1}, \ldots, x_{n} ; f\right]$ is independent of the order of $x_{1}, \ldots, x_{n}$. The function $f$ is said to be $p$-convex on $A$ if $\left[x_{1}, \ldots, x_{p+1} ; f\right] \geq 0$ for every system of distinct points $x_{1}, \ldots, x_{p+1} \in A$. For $p=0$ this means that $f$ is nonnegative on $A$. The function $f$ is 1 -convex if it is increasing on $A$; while 2-convexity coincides with the notion of usual convexity. If $A$ is an open interval and $n \geq 2$, then $f$ is $n$-convex on $A$ if and only if $f$ is $n-2$ times continuously differentiable and $f^{(n-2)}$ is convex on $A$. (See [2].)

In this note we shall investigate the validity of the following generalizations of $\left(M_{1}\right)$ and $\left(Z_{1}\right)$.

$\left(M_{n}\right)$. If the set $H=\left\{x \in[a, b]: \underline{f}_{(n)}(x)>0\right\}$ is of positive outer measure, then there is a subset $A \subset H$ such that $\lambda(A)>0$ and $f$ is n-convex on $A$.

$\left(Z_{n}\right)$. The set $\left\{x \in[a, b]: f_{(n)}(x)=\infty\right\}$ is of measure zero for every $f$ : $[a, b] \rightarrow \mathbb{R}$.

First we consider the case of $n=2$.

Proposition 1. Both $\left(M_{2}\right)$ and $\left(Z_{2}\right)$ are true.

Proof. We start with $\left(M_{2}\right)$. Suppose that $H=\left\{x \in[a, b]: \underline{f}_{(2)}(x)>0\right\}$ is of positive outer measure. Let

$$
\begin{gathered}
H_{n}=\left\{x \in H: f(y)-f(x)-f^{\prime}(x)(y-x)>0 \text { for every } y \in(a, b)\right. \\
\text { satisfying } 0<|y-x|<1 / n\} .
\end{gathered}
$$

It is clear that $H=\cup_{n=1}^{\infty} H_{n}$, and thus there is an $n$ such that $\lambda\left(H_{n}\right)>0$. Choose a subinterval $I$ of $[a, b]$ such that $|I|<1 / n$ and $\lambda\left(H_{n} \cap I\right)>0$. Let $A=H_{n} \cap I$. Then $f(y)-f(x)-f^{\prime}(x)(y-x)>0$ for every $x \in A$ and $y \in I$ with $y \neq x$. Let $x, y, z \in A, x<y<z$. Then $f(x)-f(y)-f^{\prime}(y)(x-y)>0$ and $f(z)-f(y)-f^{\prime}(y)(z-y)>0$. If we divide the first inequality by $y-x$, the second inequality by $z-y$, and add the resulting inequalities, then we obtain that the second divided difference of $f$ at the points $x, y, z$ is positive. Therefore $f$ is convex on $A$.

Now we prove $\left(Z_{2}\right)$. Suppose that $\left(Z_{2}\right)$ is false, and let $f:[a, b] \rightarrow \mathbb{R}$ be such that $E=\left\{x \in[a, b]: f_{(2)}(x)=\infty\right\}$ is of positive measure. By $\left(M_{2}\right)$, there is a set $A \subset E$ such that $\lambda(A)>0$ and $f$ is convex on $A$. We can select two points, $c, d \in A$ such that $c<d$ and $\lambda(A \cap[c, d])>0$. It is well-known that $f \mid A \cap[c, d]$ can be extended to $[c, d]$ as a convex function. It is also well-known that every convex function has a finite second Peano derivative almost everywhere. However, if $x \in A \cap(c, d)$, then $f_{(2)}(x)=\infty$ and thus no extension of $f \mid A \cap[c, d]$ can have a finite second Peano derivative at $x$. Since $\lambda(A \cap[c, d])>0$, this is a contradiction, and thus $\left(Z_{2}\right)$ is true. 
It was claimed by P. S. Bullen and S. N. Mukhopadhyay [3, (7.24) Corollary] that $\left(Z_{n}\right)$ is true for every $n$; however, their proof is in error ${ }^{1}$. In fact, as we shall see, both $\left(M_{n}\right)$ and $\left(Z_{n}\right)$ are false for every $n \geq 3$. We remark, however, that the following special case of $\left(Z_{n}\right)$ is true.

Proposition 2. If $f: A \rightarrow \mathbb{R}$ is n-convex on $A$, then the set $\{x \in A$ : $\left.f_{(n)}(x)=\infty\right\}$ is of measure zero.

We shall give the proof in the next section. It is well-known that the following stronger version of $\left(Z_{1}\right)$ is also true. The set $\left\{x \in[a, b]: f_{+}^{\prime}(x)=\infty\right\}$ is of measure zero for every $f:[a, b] \rightarrow \mathbb{R}$. (See [5, (4.4) Theorem, p. 270].) We shall prove that this statement does not generalize to $n=2$.

Theorem 3. There exists a continuously differentiable function $f$ on $[0,1]$ such that $f_{(2)+}(x)=+\infty$ and $f_{(2)-}(x)=-\infty$ holds a.e. on $[0,1]$.

For $n \geq 3$ we can prove the following.

Theorem 4. For every $n \geq 3$ there is an $n-1$ times continuously differentiable function $f$ on $[0,1]$ such that $f_{(n)}(x)=+\infty$ holds a.e. on $[0,1]$.

Corollary 5. $\left(M_{n}\right)$ and $\left(Z_{n}\right)$ are false for every $n \geq 3$.

Proof. Let $n \geq 3$. It is clear that Theorem 4 contradicts $\left(Z_{n}\right)$. Suppose that $\left(M_{n}\right)$ is true. Let $f$ be a function as in Theorem 4 . Since $E=\{x \in[a, b]$ : $\left.f_{(n)}(x)=\infty\right\}$ is of positive measure, it follows from $\left(M_{n}\right)$ that $f$ is $n$-convex on a set $A \subset E$ with $\lambda(A)>0$. By Proposition 2, the set $\left\{x \in A: f_{(n)}(x)=\infty\right\}$ is of measure zero. However, this set equals $A$, a contradiction.

We shall give the proofs of Theorems 3 and 4 in Section 3.

It is easy to see that the category analogue of $\left(Z_{n}\right)$ fails for every $n$. Indeed, let $H \subset[0,1]$ be a residual null set. It is well-known that there is an increasing continuous function $f:[0,1] \rightarrow \mathbb{R}$ such that $f^{\prime}(x)=\infty$ for every $x \in H$. That is, the set $\left\{x: f^{\prime}(x)=\infty\right\}$ can be residual. If we take the $(n-1)^{\text {st }}$ integral function of $f$, then we obtain an $n$-convex function $g$ such that the set $\left\{x \in[0,1]: g_{(n)}(x)=\infty\right\}$ is residual. We shall prove, however, that the category analogue of $\left(M_{n}\right)$ is true for every $n$.

Theorem 6. Let $f:[a, b] \rightarrow \mathbb{R}$ be such that the set $\left\{x \in[a, b]: f_{(n)}(x)>0\right\}$ is of second category. Then $f$ is n-convex on a subinterval of $[a, \bar{b}]$.

\footnotetext{
${ }^{1}$ The error appears in the proof of (7.16) Lemma on p. 274. Here the quantities $\delta_{1}$, etc. are treated as constants, while $\delta_{1}$ depends on $x_{r}, \delta_{2}$ depends on $x_{r}$ and $x_{r-1}$, etc.
} 
We shall give the proof in Section 4. Theorem 6 has the following interesting consequence.

Corollary 7. The set $E_{n}(f)=\left\{x \in[a, b]: f_{(n)}(x)=\infty\right\}$ cannot be residual and of full measure simultaneously. More precisely, if $E_{n}(f)$ is of full measure, then it is of first category.

Proof. Suppose that $E_{n}(f)$ is of full measure and of second category. By Theorem 6 , there is a subinterval $I \subset[a, b]$ such that $f$ is $n$-convex on $I$. Then the $n^{\text {th }}$ Peano derivative of $f$ is finite almost everywhere in $I$. However, $f_{(n)}(x)=\infty$ at each point of $E_{n}(f) \cap I$, which is a set of positive measure. This contradiction proves the statement.

We note that $E_{n}(f)$ can be residual and of positive measure; see Remark 9 following the proof of Theorem 4 .

\section{Proof of Proposition 2}

The statement of Proposition 2 is an immediate corollary of the following theorem by P. S. Bullen and S. N. Mukhopadhyay [3, (6.1) Theorem, p. 267]: If $f$ is n-convex on a measurable set $A$ on which $f_{(n-1)}$ exists finitely, then $f_{(n), a p}$ (the approximative $n^{\text {th }}$ Peano derivative of $f$ ) exists finitely almost everywhere on A. Unfortunately, the proof given by P. S. Bullen and S. N. Mukhopadhyay is not correct ${ }^{2}$. Actually, the proof does not use the $n$-convexity of $f$, only the fact that $f_{(n-1)}$ is increasing on $A$. Under this weaker condition the statement may fail even for $n=2$. In fact, it is easy to construct a continuously differentiable function $f$ and a perfect set $A$ of positive measure such that $f^{\prime}=0$ on $A$ (In particular, $f^{\prime}$ is increasing on $A$.), but $f_{(2), a p}$ does not exist at any point of $A$. The question, whether the statement of $[3,(6.1)$ Theorem] is true or not, remains open.

The following simple lemma is well-known (see [2]). For the sake of completeness, we give the proof.

Lemma 8. Suppose that $f$ is $n$-convex on $A$. If $\left\{x_{1}, \ldots, x_{n}\right\}$ and $\left\{y_{1}, \ldots, y_{n}\right\}$ are two sets of distinct elements of $A$ such that $x_{i} \leq y_{i}(i=1, \ldots, n)$, then $\left[x_{1}, \ldots, x_{n} ; f\right] \leq\left[y_{1}, \ldots, y_{n} ; f\right]$.

Proof. Let $x_{1}, \ldots, x_{n-1} \in A$ be distinct elements, and define a function $g$ by $g(x)=\left[x_{1}, \ldots, x_{n-1}, x ; f\right]$. Clearly $\left[x_{1}, \ldots, x_{n}, x_{n+1} ; f\right]=\left[x_{n}, x_{n+1} ; g\right]$

\footnotetext{
${ }^{2}$ The error appears in the estimate (6.7), where it is assumed that the relations (6.3) and (6.4) hold uniformly in the intervals $\left(x_{i}, x_{i}+\delta_{i}\right)$. However, the uniformity of these relations is not proved in Theorem (3.1).
} 
for every $x_{n}, x_{n+1} \in A \backslash\left\{x_{1}, \ldots, x_{n-1}\right\}$. Since $f$ is $n$-convex, it follows that every difference quotient of $g$ is nonnegative; that is, $g$ is increasing. Therefore $\left[x_{1}, \ldots, x_{n} ; f\right] \leq\left[x_{1}, \ldots, x_{n-1}, y_{n} ; f\right]$ whenever $x_{n} \leq y_{n}$. Using the fact that the divided differences do not depend on the order of the elements $x_{i}$ we find that $\left[x_{1}, \ldots, x_{n} ; f\right]$ is an increasing function of each of the variables $x_{i}$, from which the statement of the lemma is obvious.

Proof of Proposition 2. Let $f: A \rightarrow \mathbb{R}$ be $n$-convex, and put $E=$ $\left\{x \in A: f_{(n)}(x)=\infty\right\}$. We have to prove $\lambda(E)=0$. Deleting a suitable countable subset, we may assume that every point of $A$ is a bilateral point of accumulation of $A$. Let $u=\inf A$ and $v=\sup A$. It is enough to show that $\lambda(E \cap(a, b))=0$ for every $u<a<b<v$. Fix such an $a$ and $b$. Since $A$ has no isolated points, the sets $A \cap[u, a)$ and $A \cap(b, v]$ are infinite. We fix distinct elements $u_{1}, \ldots, u_{n} \in A \cap[u, a)$ and $v_{1}, \ldots, v_{n} \in A \cap(b, v]$, and put $\left[u_{1}, \ldots, u_{n} ; f\right]=L,\left[v_{1}, \ldots, v_{n} ; f\right]=M$. Then, by Lemma 8 , we have $L \leq\left[x_{1}, \ldots, x_{n} ; f\right] \leq M$ for every $x_{1}, \ldots, x_{n} \in E \cap[a, b]$.

For every interval $J \subset[a, b]$ we shall denote by $C(J)$ the convex hull of the set of numbers $\left[x_{1}, \ldots, x_{n} ; f\right]$, where $x_{1}, \ldots, x_{n}$ are arbitrary distinct elements of $A \cap J$. Then $C(J) \subset[L, M]$ for every interval $J \subset[a, b]$. It follows from Lemma 8 that if $J_{1}, J_{2}$ are disjoint subintervals of $[a, b]$, then the intervals $C\left(J_{1}\right)$ and $C\left(J_{2}\right)$ are nonoverlapping.

Let $K>0$ and let $\mathcal{U}_{K}$ be the family of all intervals $[c, d] \subset[a, b]$ for which there are elements $c=x_{1}<\cdots<x_{n+1}=d$ such that $\left[x_{1}, \ldots, x_{n+1} ; f\right]>K$. We show that $|C(J)|>K \cdot|J|$ for every $J \in \mathcal{U}_{K}$. Indeed, let $J=[c, d]$, and let $c=x_{1}<\cdots<x_{n+1}=d$ be such that $\left[x_{1}, \ldots, x_{n+1} ; f\right]>K$. Let $\left[x_{1}, \ldots, x_{n} ; f\right]=\alpha$ and $\left[x_{2}, \ldots, x_{n+1} ; f\right]=\beta$. Then by $(2)$

$$
K<\left[x_{1}, \ldots, x_{n+1} ; f\right]=\frac{\beta-\alpha}{\left(x_{n+1}-x_{1}\right)}=\frac{\beta-\alpha}{|J|} .
$$

Since $\alpha, \beta \in C(J)$, we find $|C(J)| \geq \beta-\alpha>K \cdot|J|$, as we stated.

Next we show that $\mathcal{U}_{K}$ is a Vitali cover of the set $E \cap(a, b)$. Indeed, let $x \in E \cap(a, b)$ and $\delta>0$ be given. Since $f_{(n)}(x)=\infty$, it follows from [3, (4.1) Lemma, p. 266] that

$$
\lim _{\substack{x_{n+1} \in A \\ x_{n+1} \rightarrow x}} \ldots \lim _{\substack{x_{1} \in A \\ x_{1} \rightarrow x}}\left[x_{1}, \ldots, x_{n+1} ; f\right]=\infty .
$$

Since $x$ is a bilateral point of accumulation of $A$, we may find distinct elements $x_{1}, \ldots, x_{n+1} \in A$ such that $\left[x_{1}, \ldots, x_{n+1} ; f\right]>K$ and $x-\delta<c \leq x \leq d<x+\delta$, where $c=\min _{1 \leq i \leq n+1} x_{i}$ and $d=\max _{1 \leq i \leq n+1} x_{i}$. Then $x \in[c, d] \in \mathcal{U}_{K}$ and $[c, d] \subset(x-\delta, x+\delta)$, proving that $\mathcal{U}_{K}$ is indeed a Vitali cover of $E \cap(a, b)$. By 
Vitali's covering theorem, there is a sequence $J_{i}$ of pairwise disjoint elements of $\mathcal{U}_{K}$ covering a.e. point of $E \cap(a, b)$. Now the intervals $C\left(J_{i}\right)$ are pairwise nonoverlapping and are contained in $[L, M]$. Therefore

$$
\lambda(E \cap(a, b)) \leq \sum_{i}\left|J_{i}\right|<\sum_{i}\left|C\left(J_{i}\right)\right| / K \leq(M-L) / K .
$$

Since $K$ was arbitrary, we conclude that $\lambda(E \cap(a, b))=0$.

\section{Proofs of Theorems 3 and 4}

The proofs of Theorems 3 and 4 are based on the construction of certain symmetric Cantor sets defined as follows. Let $\alpha$ and $\beta$ be real numbers satisfying $0<\beta<1$ and

$$
1-\frac{\alpha}{1-\beta}>\alpha>0
$$

We put $I_{\emptyset}=[0,1]$. Let $n \geq 0, i_{1}, \ldots, i_{n} \in\{0,1\}$, and suppose that the closed interval $I_{i_{1} \ldots i_{n}}$ has been defined such that

$$
\left|I_{i_{1} \ldots i_{n}}\right|=\frac{1}{2^{n}}\left(1-\sum_{k=0}^{n-1} \alpha \cdot \beta^{k}\right) .
$$

(The condition is satisfied for $n=0$.) Let $J_{i_{1} \ldots i_{n}}$ denote the open interval concentric with $I_{i_{1} \ldots i_{n}}$ and of length $\alpha \cdot(\beta / 2)^{n}$. Since

$$
\left|I_{i_{1} \ldots i_{n}}\right|>\frac{1}{2^{n}}\left(1-\alpha \sum_{k=0}^{\infty} \beta^{k}\right)=\frac{1}{2^{n}}\left(1-\frac{\alpha}{1-\beta}\right)>\frac{\alpha}{2^{n}}>\alpha \cdot(\beta / 2)^{n},
$$

$J_{i_{1} \ldots i_{n}}$ is a subinterval of $I_{i_{1} \ldots i_{n}}$. Let $I_{i_{1} \ldots i_{n} 0}$ and $I_{i_{1} \ldots i_{n} 1}$ denote the two components of $I_{i_{1} \ldots i_{n}} \backslash J_{i_{1} \ldots i_{n}}$. Then

$$
\left|I_{i_{1} \ldots i_{n} 0}\right|=\left|I_{i_{1} \ldots i_{n} 1}\right|=\frac{1}{2^{n+1}}\left(1-\sum_{k=0}^{n} \alpha \cdot \beta^{k}\right) .
$$

In this way we have defined $I_{i_{1} \ldots i_{n}}$ and $J_{i_{1} \ldots i_{n}}$ for every finite $0-1$ sequence $i_{1}, \ldots, i_{n}$. The intervals $J_{i_{1} \ldots i_{n}}$ are pairwise disjoint. Let $G_{\alpha, \beta}$ denote the union of the intervals $J_{i_{1} \ldots i_{n}}$, and let $P_{\alpha, \beta}=[0,1] \backslash G_{\alpha, \beta}$. Then $G_{\alpha, \beta}$ is a dense, open subset of $[0,1], P_{\alpha, \beta}$ is perfect, and

$$
\lambda\left(P_{\alpha, \beta}\right)=1-\sum_{n=0}^{\infty} \alpha \cdot \beta^{n}=1-\frac{\alpha}{1-\beta}>\alpha>0 .
$$


Proof of Theorem 3. Let $\frac{1}{2}<\beta<1$ be fixed and choose an $\alpha$ satisfying (3). Let $P=P_{\alpha, \beta}$. We define $g(x)=0$ if $x \in P, 1 /(n+1)$ if $x$ is the midpoint of $J_{i_{1} \ldots i_{n}}$, and define $g$ linearly in the closures of both halves of $J_{i_{1} \ldots i_{n}}$. Then $g$ is nonnegative and continuous on $[0,1]$. We put $\phi(x)=\int_{0}^{x} g(t) d t(x \in[0,1])$; then $\phi$ is continuously differentiable and strictly increasing on $[0,1]$.

Let $x, y \in P, x<y$ be given. There are $n \geq 0$ and $i_{1}, \ldots, i_{n}=0,1$ such that $x, y \in I_{i_{1} \ldots i_{n}}, x \in I_{i_{1} \ldots i_{n} 0}$, and $y \in I_{i_{1} \ldots i_{n} 1}$. Then $y-x \leq\left|I_{i_{1} \ldots i_{n}}\right| \leq 1 / 2^{n}$ and

$$
\phi(y)-\phi(x)=\int_{x}^{y} g(t) d t \geq \int_{J_{i_{1} \ldots i_{n}}} g(t) d t=\frac{1}{2} \cdot \alpha \cdot(\beta / 2)^{n} \cdot \frac{1}{n+1} .
$$

If $x \in P$ is fixed, $y \in P, y>x$ and $y \rightarrow x$, then $n \rightarrow \infty$ and hence

$$
\frac{\phi(y)-\phi(x)}{(y-x)^{2}} \geq \frac{1}{2} \cdot \alpha \cdot(\beta / 2)^{n} \cdot \frac{1}{n+1} \cdot 4^{n} \rightarrow \infty,
$$

as $\beta>1 / 2$. Suppose now that $x$ is a density point of $P$, and let $x_{k}>x, x_{k} \rightarrow$ $x, x_{k} \in G_{\alpha, \beta}$. If $x_{k} \in\left(a_{k}, b_{k}\right)$ where $\left(a_{k}, b_{k}\right)$ is an interval contiguous to $P$, then $\left(b_{k}-x\right) /\left(a_{k}-x\right) \rightarrow 1$ as $k \rightarrow \infty$, and hence

$$
\frac{\phi\left(x_{k}\right)-\phi(x)}{\left(x_{k}-x\right)^{2}}>\frac{\phi\left(a_{k}\right)-\phi(x)}{\left(b_{k}-x\right)^{2}}=\frac{\phi\left(a_{k}\right)-\phi(x)}{\left(a_{k}-x\right)^{2}} \cdot\left(\frac{a_{k}-x}{b_{k}-x}\right)^{2} \rightarrow \infty .
$$

Therefore $\lim _{y \rightarrow x+0} \frac{\phi(y)-\phi(x)}{(y-x)^{2}}=\infty$ for every density point $x$ of $P$. Similarly we can prove that $\lim _{y \rightarrow x-0} \frac{\phi(y)-\phi(x)}{(y-x)^{2}}=-\infty$ for every density point $x$ of $P$. Summing up, we constructed a function $\phi$ with the following properties.

(i) $\phi$ is continuously differentiable on $[0,1]$;

(ii) $\phi$ is strictly increasing on $[0,1]$;

(iii) $\phi^{\prime}(x)=0$ if $x \in P$;

(iv) $\phi$ is twice differentiable a.e. in $[0,1] \backslash P$;

(v) $\lim _{y \rightarrow x \pm 0} \frac{\phi(y)-\phi(x)}{(y-x)^{2}}= \pm \infty$ if $x$ is a density point of $P$.

(As for (iv), note that $\phi$ is locally a quadratic polynomial at each point of $[0,1] \backslash P$ apart from the midpoints of the intervals contiguous to $P$.)

Let $\mathcal{P}$ denote the family of all subsets of $[0,1]$ that are homothetic with $P$. Then $\mathcal{P}$ is a Vitali cover of $[0,1]$, and thus there is a sequence $\left(P_{k}\right)$ of 
pairwise disjoint elements of $\mathcal{P}$ that cover a.e. point of $[0,1]$ (see $[5$, Chapter IV, Theorem (3.1), p. 109]). Let $c_{k}=\min P_{k}$ and $d_{k}=\max P_{k}(k=1,2, \ldots)$. We define

$$
f_{k}(x)= \begin{cases}\frac{d_{k}-c_{k}}{2^{k}} \phi\left(\frac{x-c_{k}}{d_{k}-c_{k}}\right) & \text { if } x \in\left[0, c_{k}\right] \\ \frac{d_{k}-c_{k}}{2^{k}} \phi(1) & \text { if } x \in\left[c_{k}, d_{k}\right]\end{cases}
$$

for every $k$. Then $f_{k}$ is continuously differentiable on [0,1]. Also,

$$
0 \leq f_{k}(x) \leq \frac{1}{2^{k}} \phi(1) \text { and } 0 \leq f_{k}^{\prime}(x) \leq \frac{1}{2^{k}} \max _{t \in[0,1]} \phi^{\prime}(t)=\frac{1}{2^{k}}
$$

for every $x \in[0,1]$. We put $f=\sum_{k=1}^{\infty} f_{k}$. Then $f$ is continuously differentiable on $[0,1]$ and $f^{\prime}=\sum_{k=1}^{\infty} f_{k}^{\prime}$.

We claim that almost every $x \in[0,1]$ has the following properties.

(vi) There is a $k_{1}=k_{1}(x)$ such that $x$ is a density point of $P_{k_{1}}$;

(vii) There is a $k_{2}=k_{2}(x)$ such that $x \notin\left[c_{k}, d_{k}\right]$ for every $k>k_{2}$; and

(viii) If $k$ is such that $x \notin P_{k}$, then $f_{k}$ is twice differentiable at $x$.

Indeed, (vi) is obvious from the fact that $\cup_{k=1}^{\infty} P_{k}$ covers a.e. point of $[0,1]$. In order to prove (vii), note that each $P_{k}$ is homothetic with $P$, and thus $\lambda\left(P_{k}\right) /\left(d_{k}-c_{k}\right)=\lambda(P)$ for every $k$. Therefore

$$
\sum_{k=1}^{\infty}\left(d_{k}-c_{k}\right)=\sum_{k=1}^{\infty} \frac{d_{k}-c_{k}}{\lambda\left(P_{k}\right)} \lambda\left(P_{k}\right)=\frac{1}{\lambda(P)} \sum_{k=1}^{\infty} \lambda\left(P_{k}\right)=\frac{1}{\lambda(P)}<\infty,
$$

and thus the set of points contained by infinitely many of the intervals $\left[c_{k}, d_{k}\right]$ is null. Finally, (viii) is immediate from (iv). We shall complete the proof by showing that if a point $x \in[0,1]$ satisfies (vi)-(viii), then $f_{(2) \pm}(x)= \pm \infty$. Let $x$ be such a point, and put

$$
\frac{f_{k}(y)-f_{k}(x)-f_{k}^{\prime}(x)(y-x)}{(y-x)^{2}}=A_{k}(y)
$$

for every $k=1,2, \ldots$ and $y>x$. Let $k_{1}$ and $k_{2}$ be as in (vi) and (vii). If $k>k_{2}$, then $x \notin\left[c_{k}, d_{k}\right]$ and thus $f_{k}^{\prime}(x)=0$. Since $f_{k}$ is increasing, we find that $A_{k}(y) \geq 0$ for every $k>k_{2}$ and $y>x$. Let $k_{3}=\max \left(k_{1}, k_{2}\right)$. Then for every $y>x$ we have

$$
\frac{f(y)-f(x)-f^{\prime}(x)(y-x)}{(y-x)^{2}}=\sum_{k=1}^{\infty} A_{k}(y) \geq \sum_{k=1}^{k_{3}} A_{k}(y)
$$


Since $x$ is a density point of $P_{k_{1}}$, we have

$$
\lim _{y \rightarrow x+0} A_{k_{1}}(y)=\lim _{y \rightarrow x+0} \frac{f_{k_{1}}(y)-f_{k_{1}}(x)}{(y-x)^{2}}=\infty .
$$

If $k \neq k_{1}$, then $x \notin P_{k}$ and thus, by (viii), $f_{k}$ is twice differentiable at $x$ and $\lim _{y \rightarrow x+0} A_{k}(y)=f_{k}^{\prime \prime}(x) / 2$. Therefore, by (4) we obtain

$$
\liminf _{y \rightarrow x+0} \frac{f(y)-f(x)-f^{\prime}(x)(y-x)}{(y-x)^{2}} \geq \sum_{\substack{k \leq k_{3} \\ k \neq k_{1}}} \frac{f_{k}^{\prime \prime}(x)}{2}+\liminf _{y \rightarrow x+0} A_{k_{1}}(y)=\infty,
$$

and thus $f_{(2)+}(x)=+\infty$. Similar argument gives $f_{(2)-}(x)=-\infty$.

Proof of TheOREM 4. It is enough to construct a twice continuously differentiable function $f$ such that $f_{(3)}=\infty$ a.e. Indeed, if we take the $(n-3)^{\mathrm{rd}}$ integral function of $f$, then we obtain a function satisfying the requirements of the theorem.

Let $\frac{1}{\sqrt{2}}<\beta<1$ be fixed, and choose an $\alpha$ satisfying (3). Let $P=P_{\alpha, \beta}$. We define a function $g$ as follows. First, if $x \in P$, then we put $g(x)=0$. Next we define $g$ on the intervals contiguous to $P$. Let $J_{i_{1} \ldots i_{n}}=(a, b)$ be such an interval, and let $c=a+(b-a) / 3$ and $d=a+2(b-a) / 3$. We put $g(c)=1 /(n+1), g(d)=-1 /(n+1)$, and let $g$ be linear on each of the closed intervals $[a, c],[c, d]$, and $[d, b]$. In this way we defined $g$ on $[0,1]$. It is easy to see that $g$ is continuous.

Let $h(x)=\int_{0}^{x} g(t) d t(x \in[0,1])$. Then $h$ is continuously differentiable on $[0,1]$. Since the integral of $g$ over each interval contiguous to $P$ equals zero, it follows that $h(x)=0$ if $x \in P$. It is easy to see that $h(x)>0$ if $x \in[0,1] \backslash P$. It is also easy to check that

$$
\int_{J_{i_{1} \ldots i_{n}}} h(x) d x=\frac{1}{9(n+1)}\left|J_{i_{1} \ldots i_{n}}\right|^{2}=\frac{\alpha^{2}}{9(n+1)} \cdot(\beta / 2)^{2 n} .
$$

Now we put $\phi(x)=\int_{0}^{x} h(t) d t(x \in[0,1])$. Then $\phi$ is twice continuously differentiable and $\phi^{\prime \prime}=g$.

Let $x, y \in P, x<y$ be given. There are $n \geq 0$ and $i_{1}, \ldots, i_{n}=0,1$ such that $x, y \in I_{i_{1} \ldots i_{n}}, x \in I_{i_{1} \ldots i_{n} 0}$, and $y \in I_{i_{1} \ldots i_{n} 1}$. Then $y-x \leq\left|I_{i_{1} \ldots i_{n}}\right| \leq 1 / 2^{n}$ and

$$
\phi(y)-\phi(x)=\int_{x}^{y} h(t) d t \geq \int_{J_{i_{1} \ldots i_{n}}} h(t) d t=\frac{\alpha^{2}}{9(n+1)} \cdot(\beta / 2)^{2 n} .
$$

Therefore

$$
\frac{\phi(y)-\phi(x)}{(y-x)^{3}} \geq \frac{\alpha^{2}}{9(n+1)} \cdot(\beta / 2)^{2 n} \cdot 8^{n} \rightarrow \infty
$$


if $y \rightarrow x+0$ and $y \in P$. The same is true if $y \rightarrow x-0$ and $y \in P$. If $x$ is a density point of $P$, then in a similar way as in the proof of Theorem 3 we conclude that $\lim _{y \rightarrow x} \frac{\phi(y)-\phi(x)}{(y-x)^{3}}=\infty$.

Summing up, we constructed a function $\phi$ with the following properties.

(ix) $\phi$ is twice continuously differentiable on $[0,1]$;

(x) $\phi$ is strictly increasing on $[0,1]$;

(xi) $\phi^{\prime}(x)=\phi^{\prime \prime}(x)=0$ if $x \in P$;

(xii) $\phi$ is three times differentiable a.e. in $[0,1] \backslash P$;

(xiii) $\lim _{y \rightarrow x} \frac{\phi(y)-\phi(x)}{(y-x)^{3}}=\infty$ if $x$ is a density point of $P$.

Now we repeat the argument of the proof of Theorem 3 . Let $P_{k}, c_{k}, d_{k}$ be as in the proof of Theorem 3. We define

$$
f_{k}(x)= \begin{cases}0 & \text { if } x \in\left[0, c_{k}\right], \\ \frac{\left(d_{k}-c_{k}\right)^{2}}{2^{k}} \phi\left(\frac{x-c_{k}}{d_{k}-c_{k}}\right) & \text { if } x \in\left[c_{k}, d_{k}\right], \\ \frac{\left(d_{k}-c_{k}\right)^{2}}{2^{k}} \phi(1) & \text { if } x \in\left[d_{k}, 1\right]\end{cases}
$$

for every $k$. Then $f_{k}$ is continuously differentiable on $[0,1]$, and

$$
0 \leq f_{k}(x) \leq \frac{1}{2^{k}} \phi(1), \quad 0 \leq f_{k}^{\prime}(x) \leq \frac{1}{2^{k}} \quad \text { and } \quad 0 \leq f_{k}^{\prime \prime}(x) \leq \frac{1}{2^{k}}
$$

for every $x \in[0,1]$. We put $f=\sum_{k=1}^{\infty} f_{k}$. Then $f$ is twice continuously differentiable on $[0,1], f^{\prime}=\sum_{k=1}^{\infty} f_{k}^{\prime}$ and $f^{\prime \prime}=\sum_{k=1}^{\infty} f_{k}^{\prime \prime}$.

Suppose that a point $x \in[0,1]$ satisfies (vi) and (vii) (See the proof of Theorem 3.) as well as the following property.

(xiv) If $k$ is such that $x \notin P_{k}$, then $f_{k}$ is three times differentiable at $x$.

We shall prove that in this case $f_{(3)}(x)=\infty$. Since (vi), (vii) and (xiv) hold for a.e. point, this will complete the proof. Let $x$ be a point satisfying (vi), (vii) and (xiv), and for every $k=1,2, \ldots$ and $y \neq x$ put

$$
\frac{f_{k}(y)-f_{k}(x)-f_{k}^{\prime}(x)(y-x)-\frac{f_{k}^{\prime \prime}(x)}{2}(y-x)^{2}}{(y-x)^{3}}=B_{k}(y) .
$$


Let $k_{1}$ and $k_{2}$ be as in (vi) and (vii). If $k>k_{2}$, then $x \notin\left[c_{k}, d_{k}\right]$ and thus $f_{k}^{\prime}(x)=f_{k}^{\prime \prime}(x)=0$. Since $f_{k}$ is increasing, we find that $B_{k}(y) \geq 0$ for every $k>k_{2}$ and $y \neq x$. Let $k_{3}=\max \left(k_{1}, k_{2}\right)$. Then for every $y \neq x$ we have

$$
\frac{f(y)-f(x)-f^{\prime}(x)(y-x)-\frac{f^{\prime \prime}(x)}{2}(y-x)^{2}}{(y-x)^{3}}=\sum_{k=1}^{\infty} B_{k}(y) \geq \sum_{k=1}^{k_{3}} B_{k}(y) .
$$

Since $x$ is a density point of $P_{k_{1}}$, we have

$$
\lim _{y \rightarrow x} B_{k_{1}}(y)=\lim _{y \rightarrow x} \frac{f_{k_{1}}(y)-f_{k_{1}}(x)}{(y-x)^{3}}=\infty .
$$

If $k \neq k_{1}$, then $x \notin P_{k}$ and thus, by (xiv), $f_{k}$ is three times differentiable at $x$ and $\lim _{y \rightarrow x} B_{k}(y)=f_{k}^{\prime \prime \prime}(x) / 6$. Therefore, by (5) we obtain

$$
\begin{gathered}
\liminf _{y \rightarrow x} \frac{f(y)-f(x)-f^{\prime}(x)(y-x)-\frac{f^{\prime \prime}(x)}{2}(y-x)^{2}}{(y-x)^{3}} \\
\geq \sum_{\substack{k \leq k_{3} \\
k \neq k_{1}}} \frac{f_{k}^{\prime \prime \prime}(x)}{6}+\liminf _{y \rightarrow x} B_{k_{1}}(y)=\infty
\end{gathered}
$$

and thus $f_{(3)}(x)=\infty$.

Remark 9. The function $\phi$ constructed in the proof of Theorem 4 satisfies (ix)-(xiii), and thus $\phi_{(3)}(x)=\infty$ holds at each density point $x$ of $P$. Let $d(x)$ denote the distance of $x$ from $P$. It is clear that whenever a function $\psi$ satisfies $\phi \leq \psi \leq \phi+d^{3}$ on $[0,1]$, then $\psi_{(3)}(x)=\infty$ at each density point $x$ of $P$. As we mentioned in the introduction, there is a continuous function $\alpha$ on $[0,1]$ such that $\alpha_{(3)}(x)=\infty$ holds at the points of a residual set. It is easy to see that sticking together suitable affine copies of the graph of $\alpha$ we can construct a function $\psi$ satisfying $\phi \leq \psi \leq \phi+d^{3}$. This function has the property that $E_{3}(\psi)$ is residual (because it is residual in each interval contiguous to $P$ ), and of positive measure (because it contains the density points of $P$ ).

\section{Proof of Theorem 6}

In the proof of Theorem 6 we shall apply some ideas of [1, Section 3].

Lemma 10. Let $f$ be locally bounded and right continuous in $(c, d)$, and let $\mu$ be a finite Borel measure on $[0,1]$ such that $\mu([1-\delta, 1])>0$ for every $\delta>0$. Suppose that $A$ is a dense subset of $(c, d)$, and for every $x \in A$ there is a real number $c(x)$ such that $\int_{0}^{1}[f(x+t h)-f(x)-c(x) t h] d \mu(t) \geq 0$ for every $h \in(c-x, d-x)$. Then $f$ is convex in $(c, d)$. 
Proof. We may assume that $d-c \leq 1$. Let $C$ denote the set of points of continuity of $f$. Since $f$ is right continuous, $(c, d) \backslash C$ is countable. In particular, $C$ is dense in $(c, d)$. It is enough to show that $f$ is convex on $C$; since $f$ is right continuous, this will imply that $f$ is convex in $(c, d)$.

Suppose $f$ is not convex on $C$. Then there are points $u, v, w \in C, u<v<w$ such that $f(v)$ lies above the chord joining $(u, f(u))$ and $(w, f(w))$. Subtracting a linear function from $f$ we may assume that $f(u)=f(w)=0$ and $f(v)>0$. Let $m=\sup \{f(x): x \in[u, w]\}$. Then $m \geq f(v)>0$. Since $f$ is locally bounded, we have $m<\infty$. Let $\delta>0$ be such that $f(x)<m / 4$ for every $x \in[u, u+\delta] \cup[w-\delta, w]$. Let $0<\eta<m / 4$ be fixed. There is a point $x_{0} \in(u, w)$ such that $f\left(x_{0}\right)>m-\eta$. Since $A$ is dense in $(u, w)$ and $f$ is right continuous at $x_{0}$, it follows that there is a point $x \in(u, w) \cap A$ such that $f(x)>m-\eta$. We show that choosing $\eta$ small enough, both $c(x) \geq 0$ and $c(x) \leq 0$ provide a contradiction.

Suppose $c(x) \geq 0$, and let $h=w-x$. Then $0<h<1$, and

$$
\begin{aligned}
0 & \leq \int_{0}^{1}[f(x+t h)-f(x)-c(x) t h] d \mu(t) \\
& \leq \int_{0}^{1}[f(x+t h)-f(x)] d \mu(t)=\int_{0}^{1-\delta}+\int_{1-\delta}^{1}=I_{1}+I_{2} .
\end{aligned}
$$

If $t \in[0,1-\delta]$, then $f(x+t h)-f(x) \leq m-(m-\eta)=\eta$ and therefore $I_{1} \leq$ $\eta \cdot \mu([0,1])$. If $t \in[1-\delta, 1]$, then $x+t h \in[w-\delta, w]$ and hence $f(x+t h)<m / 4$ and

$$
I_{2}<\left[\frac{m}{4}-(m-\eta)\right] \mu([1-\delta, 1])<-\frac{m}{2} \mu([1-\delta, 1]) .
$$

Thus if $\eta<\frac{m}{2} \cdot \frac{\mu([1-\delta, 1])}{\mu([0,1])}$, then

$$
I_{1}+I_{2}<\eta \cdot \mu([0,1])-(m / 2) \mu([1-\delta, 1])<0 .
$$

If $c(x) \leq 0$, then take $h=u-x<0$ to obtain a similar contradiction.

Lemma 11. Suppose $f$ is $k$ times differentiable in a neighborhood of $x$, and the Peano derivative $f_{(k+1)}(x)$ exists. If the right hand derivative $\left(f^{(k)}\right)_{+}^{\prime}$ of $f^{(k)}$ exists at $x$, then $\left(f^{(k)}\right)_{+}^{\prime}(x)=f_{(k+1)}(x)$.

Proof. By $k$ applications of L'Hôpital's rule we obtain

$$
\begin{aligned}
f_{(k+1)}(x) & =\lim _{t \rightarrow+0} \frac{(k+1) !}{t^{k+1}}\left(f(x+t)-\sum_{i=0}^{k} \frac{f^{(i)}(x)}{i !} t^{i}\right) \\
& =\lim _{t \rightarrow+0} \frac{f^{(k)}(x+t)-f^{(k)}(x)}{t}=\left(f^{(k)}\right)_{+}^{\prime}(x) .
\end{aligned}
$$


Proof of Theorem 6 . The proof is by induction on $n$. Let $n=1$, and suppose that the set $H=\left\{x \in[a, b]: f_{(1)}(x)>0\right\}$ is of second category. Let $H_{k}=\{x \in H:(f(y)-f(x)) /(y-\bar{x})>0$ for every $0<|y-x|<1 / k\}$. Since $H=\cup_{k=1}^{\infty} H_{k}$, there is an index $k$ such that $H_{k}$ is dense in a subinterval $I \subset[a, b]$ with $|I|<1 / k$. It is clear that $f$ is increasing (=1-convex) in $I$.

Let $n \geq 1$ and suppose that the statement is true for $n$. Let $f:[a, b] \rightarrow \mathbb{R}$ be such that the set $H=\left\{x \in[a, b]: f_{(n+1)}(x)>0\right\}$ is of second category. Since $f_{(n)}(x)$ exists and is finite for every $x \in H$, there exists a positive integer $K$ such that $f_{(n)}(x)>-K$ at the points of a second category subset of $H$. Adding $K \cdot x^{n} / n$ ! to $f$ (which does not affect the value of $f_{(n+1)}$ or the $(n+1)$-convexity of $f$ in any interval), we may assume that $f_{(n)}(x)>0$ and $f_{(n+1)}(x)>0$ at each point of $H$. Let $I$ be a subinterval of $[a, b]$ such that $H$ is of the second category in each subinterval of $I$ (see $[4, \S 10, \mathrm{~V}$. p. 85]). By the induction hypothesis, $f$ is $n$-convex in a subinterval $J \subset I$. Let $H_{k}$ denote the set of points $x \in H \cap J$ such that

$$
\frac{(n+1) !}{h^{n+1}}\left(f(x+h)-\sum_{i=0}^{n} \frac{f_{(i)}(x)}{i !} h^{i}\right)>0
$$

for every $0<|h|<1 / k$. Since $H \cap J=\cup_{k=1}^{\infty} H_{k}$, there is a $k$ such that $H_{k}$ is of the second category. Let $(c, d)$ be a subinterval of $J$ such that $d-c<1 / k$ and $H_{k}$ is dense in $(c, d)$. Putting $A=(c, d) \cap H_{k}$ we find that

(i) $f$ is $n$-convex in $(c, d)$, and

(ii) at each point of the dense subset $A \subset(c, d)$ the Peano derivative $f_{(n)}(x)$ exists finitely, and (6) holds for every $h \neq 0$ such that $x+h \in(c, d)$.

We shall prove that $f$ is $(n+1)$-convex in $(c, d)$. First suppose $n=1$. Then $f$ is increasing by (i). If $x \in A$, then $f(x+h)-f(x)-f^{\prime}(x) h>0$ for every $h \in(c-x, d-x), h \neq 0$. As we saw in the proof of Proposition 1, this implies that $f$ is convex on $A$. Since $f$ is increasing, we conclude that $f$ is convex in $(c, d)$.

Next suppose $n>1$. Since $f$ is $n$-convex in $(c, d), f$ is $n-2$ times differentiable and $f^{(n-2)}$ is convex in $(c, d)$. Then the right hand side derivative $\left(f^{(n-2)}\right)_{+}^{\prime}=g$ exists everywhere, and is increasing and right continuous in $(c, d)$. It follows from Lemma 11 that $f_{(n-1)}(x)=g(x)$ at each point of $A$. Since $f^{(n-2)}$ is convex, it is absolutely continuous, and thus $f^{(n-2)}$ equals the integral function of $g$. We shall prove that $g$ is convex in $(c, d)$. This will complete the proof. Indeed, if $g$ is continuous, then $f^{(n-2)}$ is a primitive of $g$. Therefore $f$ is $n-1$ times differentiable and $f^{(n-1)}=g$ is convex in $(c, d)$; that is, $f$ is $(n+1)$-convex in $(c, d)$. 
It is enough to show that $g$ satisfies the conditions of Lemma 10. Since $g$ is increasing, it is locally bounded in $(c, d)$. If $x \in A$, then by (ii), we have

$$
\frac{(n+1) !}{h^{n+1}}\left(f(x+h)-\sum_{i=0}^{n-2} \frac{f^{(i)}(x)}{i !} h^{i}-\frac{g(x)}{(n-1) !} h^{n-1}-\frac{f_{(n)}(x)}{n !} h^{n}\right)>0
$$

for every $h \neq 0$ such that $x+h \in(c, d)$. It is well-known (and easy to prove by induction on $k$ ) that if $f^{(k-1)}$ is absolutely continuous on $[x, x+h]$, then

$$
f(x+h)-\sum_{i=0}^{k-1} \frac{f^{(i)}(x)}{i !} h^{i}=\frac{h^{k}}{(k-1) !} \int_{0}^{1} f^{(k)}(x+t h)(1-t)^{k-1} d t .
$$

Therefore, by (7), we have

$$
\begin{aligned}
0 & <\frac{(n+1) !}{h^{n+1}}\left(\frac{h^{n-1}}{(n-2) !} \int_{0}^{1} g(x+t h)(1-t)^{n-2} d t-\frac{g(x)}{(n-1) !} h^{n-1}-\frac{f_{(n)}(x)}{n !} h^{n}\right) \\
& =\frac{(n+1) n(n-1)}{h^{2}}\left(\int_{0}^{1} g(x+t h)(1-t)^{n-2} d t-\frac{g(x)}{(n-1)}-\frac{f_{(n)}(x)}{n(n-1)} h\right) \\
& =\frac{(n+1) n(n-1)}{h^{2}} \int_{0}^{1}\left[g(x+t h)-g(x)-f_{(n)}(x) t h\right](1-t)^{n-2} d t
\end{aligned}
$$

for every $h \in(c-x, d-x), h \neq 0$. Putting $\mu(B)=\int_{B}(1-t)^{n-2} d t$, we can see that the conditions of Lemma 10 are satisfied with $f=g$. Therefore $g$ is convex on $(c, d)$, which completes the proof.

\section{References}

[1] S. Agronsky, A. M. Bruckner, M. Laczkovich and D. Preiss, Convexity conditions and intersections with smooth functions, Trans. Amer. Math. Soc. 289 (1985), 659-677.

[2] P. S. Bullen, A criterion of n-convexity, Pacific J. Math. 36 (1971), 81-98.

[3] P. S. Bullen and S. N. Mukhopadhyay, Relations between some general nth-order derivatives, Fund. Math. 85 (1974), 257-276.

[4] K. Kuratowski, Topology, Vol. I. Academic Press, 1966.

[5] S. Saks, Theory of the Integral. Dover, 1964. 
